\title{
L'agriculture biologique, un outil de développement de la filière dattes dans la région des Ziban en Algérie
}

\author{
Salah Eddine Benziouche* \\ Département des sciences agronomiques, université de Biskra, BP 145 RP, 07000 Biskra, Algérie
}

\begin{abstract}
Résumé - Les difficultés de commercialisation entravent le bon fonctionnement de la filière dattes en Algérie, particulièrement pour les cultivars à faible valeur marchande. Afin de tirer profit des expériences extérieures, et notamment de celles des agriculteurs des pays européens et des pays voisins (Maroc et Tunisie), de nombreux mécanismes et outils ont été introduits en Algérie. Nous montrons ici comment le recours à la production des dattes biologiques peut être un instrument d'amélioration de la commercialisation et de promotion des exportations des dattes algériennes, ainsi que de protection et de sauvegarde des palmeraies. Cette étude, basée sur des enquêtes de terrain, révèle que l'application des techniques de l'agriculture biologique à la phoeniciculture dans la région des Ziban, reste sous-exploitée et mal organisée, même si des résultats positifs sont enregistrés. Tous les indicateurs analysés demeurent peu intéressants jusqu'à maintenant et ce, en dépit des opportunités, nombreuses et encourageantes, qui pourraient être exploitées. En effet, le système de production de dattes biologiques ne pourra pas réussir sans la résolution de contraintes qui sont d'ordre technique, socioéconomique et de politique agricole.
\end{abstract}

Mots clés : agriculture biologique / datte / Ziban / Algérie / contraintes / valorisation

\begin{abstract}
Organic agriculture, a development tool for the date value chain in the Ziban region of Algeria. Difficulties in marketing hamper the smooth functioning of the date value chain in Algeria, especially for cultivars of low market value. In order to take advantage of the experiences of others, especially farmers from European countries and from neighboring countries (Morocco and Tunisia), many mechanisms and tools have been introduced in Algeria. In this work we show how the production of organic dates could be an instrument for improving marketing and exporting of Algerian dates, and for protecting and preserving palm groves. This study is based on field surveys. It shows that the use of organic practices in date palm cultivation in the Ziban region remains under-exploited and unorganized, although the positive results recorded. Nevertheless, all the analyzed indicators remain poor to date, despite the many and encouraging opportunities that could be exploited. Date palm organic farming will indeed not be successful without coping with the existing technical, socio-economic and political constraints.
\end{abstract}

Keywords: organic agriculture / date palm / Ziban / Algeria / constraints / valorization

\section{Introduction}

Plusieurs études (Hadjou et al., 2013 ; Rached et al., 2012) ont démontré que l'agriculture biologique $(\mathrm{AB})$ a connu un fort développement dans le monde à travers l'accroissement de la superficie destinée aux cultures biologiques (ou «bio»), l'augmentation des consommateurs du bio et de la gamme des produits bio, mais surtout l'augmentation de la taille des marchés et du chiffre d'affaires obtenus en ayant recours à ce label. Le marché mondial du bio représente actuellement plus de 80 milliards de dollars (Mercier, 2015). La réussite de l'AB

\footnotetext{
*Auteur de correspondance : Sbenziouche@yahoo.fr
}

est aussi un outil de développement des filières agricoles et de développement local dans plusieurs régions du monde. En France, l'essor de l'AB est le plus important dans le monde (Cardona, 2014 ; Peigné, 2015). D'après Caplat (2012), celleci a permis de réduire considérablement l'endettement des petits paysans, de maintenir une population agricole en place, mais aussi de renforcer les liens sociaux par la reconnaissance des savoirs traditionnels. En outre, elle a renforcé le lien avec les consommateurs et le territoire. Au Brésil, l'AB est l'un des principaux moteurs de croissance de l'agriculture ces dernières années (Georges et Blanc, 2012).

L'Algérie est l'un des grands pays leaders dans le domaine de la culture du palmier dattier, avec plus de 18 millions de palmiers et plus de 800 variétés, dont les plus importantes sont 
Deglet Nour, Ghars et Mech Degla (Benziouche et Cheriet, 2012) ; elle se classe après les Émirats Arabes Unis, l'Arabie saoudite et l'Irak (respectivement $40 ; 28,5$ et 21 millions de palmiers), mais avant l'Égypte, la Tunisie et le Maroc (respectivement 15,$5 ; 5,5$ et 4,5 millions de pieds). Beaucoup d'études ont montré la difficulté du bon fonctionnement de la filière dattes en aval (difficultés de commercialisation), notamment sur le marché international (Belguedj, 2004; Benziouche, 2012). Cela conduit parfois à un phénomène d'érosion génétique et peut entraîner l'abandon des anciennes palmeraies. Tirant profit des expériences extérieures, de nombreux mécanismes sont appliqués ou en cours de mise en place en Algérie, pour la promotion des exportations et la valorisation des dattes, comme des labels «produits de terroir » et «agriculture biologique» (AB).

La région des Ziban est la région phoenicicole la plus importante d'Algérie, en quantité et en qualité, grâce à la variété Deglet Nour. L'idée de développer la production des dattes bio a commencé dans cette région depuis une décennie, mais d'une manière très timide à travers quelques initiatives individuelles sous l'influence de certains exportateurs installés en Europe.

L'opération a connu, depuis, une expansion sur plusieurs communes de la wilaya de Biskra. Nous essayerons de montrer comment la culture des dattes biologiques pourrait être un outil d'amélioration de la commercialisation et de promotion des exportations des dattes algériennes mais aussi un moyen de protection des palmeraies contre l'érosion génétique.

Pour ce faire, nous allons :

- présenter l'état des lieux de la filière des dattes biologiques au niveau de la région des Ziban (wilaya de Biskra);

- déterminer les difficultés qui entravent l'extension de la filière bio ;

- essayer d'évaluer les impacts de l'AB sur le développement de la filière datte et des oasis, tout en mettant en relief les opportunités de l'AB dans cette région.

\section{Matériels et méthodes}

L'étude a été conduite en 2015 dans la wilaya de Biskra (Fig. 1). Le choix de l'étude des dattes bio dans cette région s'explique par l'importance phoenicicole de la zone, tant en quantité qu'en qualité. Ce choix a aussi été dicté par l'importance de la production des dattes bio dans la région, aussi bien en termes de superficie que de chiffre d'affaires.

\subsection{Enquête auprès des acteurs de la filière}

Pour recueillir les données nécessaires, et en l'absence de données préexistantes, nous nous sommes basés sur des enquêtes de terrain réalisées auprès d'acteurs de la filière dattes bio. Nous avons effectué une enquête individuelle (fin 2015) par questionnaire auprès d'une dizaine de producteurs certifiés bio ( $50 \%$ du total des 20 producteurs certifiés bio), d'une dizaine de producteurs non certifiés $(25 \%$ du total estimé à 40) et d'une trentaine de phoeniciculteurs ordinaires afin de faire une comparaison et d'expliquer le faible recours à l'agriculture bio dans la région. Le choix des producteurs est basé sur deux critères de stratification : le nombre de palmiers et la pratique du bio. Le contenu des enquêtes porte sur la situation de l'AB, les atouts, les contraintes, la maîtrise et les perspectives. Afin de disposer d'une information globale sur les pratiques culturales, nous avons réalisé des interviews qui ont porté sur les normes d'application avec des spécialistes de la question (chercheurs et vulgarisateurs) et une recherche bibliographique pour, d'une part, renforcer et argumenter l'analyse et, ensuite, pour cadrer théoriquement le sujet de recherche. Une autre enquête a été également réalisée auprès de quelques exportateurs de dattes bio; elle a porté sur le processus d'exportation, les contraintes et les prix. Certaines variables (superficie, production, exportation) ont été comparées en utilisant les références bibliographiques pertinentes.

\subsection{Indicateurs technico-économiques de la phoeniciculture dans les Ziban}

Les statistiques agricoles disponibles en 2015 montrent que le patrimoine phoenicicole de Biskra (Fig. 1) a connu une forte augmentation, passant de 2 millions de pieds en 1990 à 4,28 millions en $2015(+228 \%)$, dont $91 \%$ sont en production (Fig. 2) (DSA Biskra, 2016). Plus de $93 \%$ de cet accroissement s'explique par les plantations nouvelles encouragées par le programme d'Accession à la propriété foncière agricole (APFA) et le Programme national de développement agricole (PNDA) (Bouguedoura et al., 2015).

Le patrimoine phoenicicole algérien est réparti sur 17 des 48 wilayas du pays et se concentre principalement dans la région sud-est. La première place est occupée par la wilaya de Biskra, avec plus $25 \%$ du patrimoine national (Fig. 2). Sur le plan variétal, en 2015,61\% des palmiers de la wilaya sont de la variété Deglet Nour ; viennent ensuite les dattes sèches et assimilées (26\%) et la variété Ghars et assimilées (13\%) (DSA Biskra, 2016). En termes de production, la wilaya de Biskra occupe aussi la première place au plan national, avec $41 \% \mathrm{du}$ volume (Fig. 3).

La production de dattes de Biskra est variable selon les années ; elle est étroitement corrélée avec le nombre de palmiers en production, l'alternance biologique des palmiers qui fait qu'une bonne récolte est souvent suivie d'une mauvaise (Benziouche et Chehat, 2010), la conduite culturale ainsi que les aléas climatiques (Benziouche et Cheriet, 2012). Cette production a connu un grand essor, passant de $60000 \mathrm{t}$ en 1990 à $370000 \mathrm{t}$ en 2015, soit une hausse de $310 \%$. Quatrevingt-neuf pour cent de cette variation de production s'explique par l'entrée en production de nouvelles plantations, mais aussi par l'importance accordée à ce secteur par l'État (Benziouche, 2012). La majeure partie de la production est de type Deglet Nour $(63,5 \%)$.

Les rendements nationaux ne dépassent pas $50 \mathrm{~kg} /$ palmier en 2015. Les meilleurs rendements sont enregistrés dans les oasis de Biskra avec une moyenne de $61 \mathrm{~kg} /$ pied. Les meilleurs producteurs de la variété Deglet Nour récoltent jusqu'à $150 \mathrm{~kg} /$ arbre à Tolga (Biskra). D'après Benziouche (2012), les spécificités édaphiques et climatiques, la conduite culturale ainsi que la valeur des variétés expliquent cette importance de la production et des rendements dans la région.

Ces indicateurs technico-économiques montrent l'importance de la phoeniciculture dans la wilaya de Biskra. Dans ce contexte, l'AB représente une occasion de saisir les 


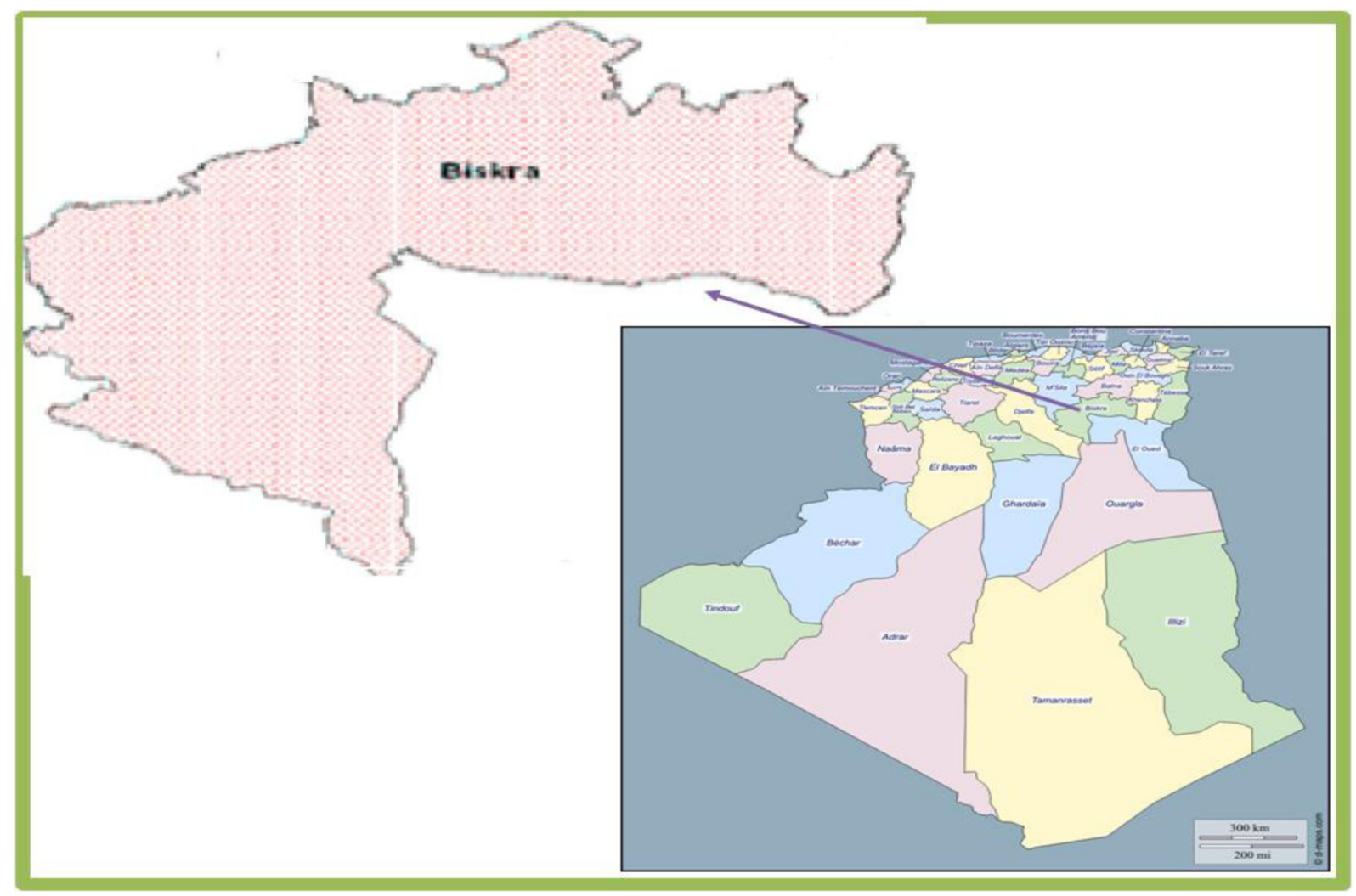

Fig. 1. Localisation de la région d'étude (Biskra).

Fig. 1. Map of the study area (Biskra).

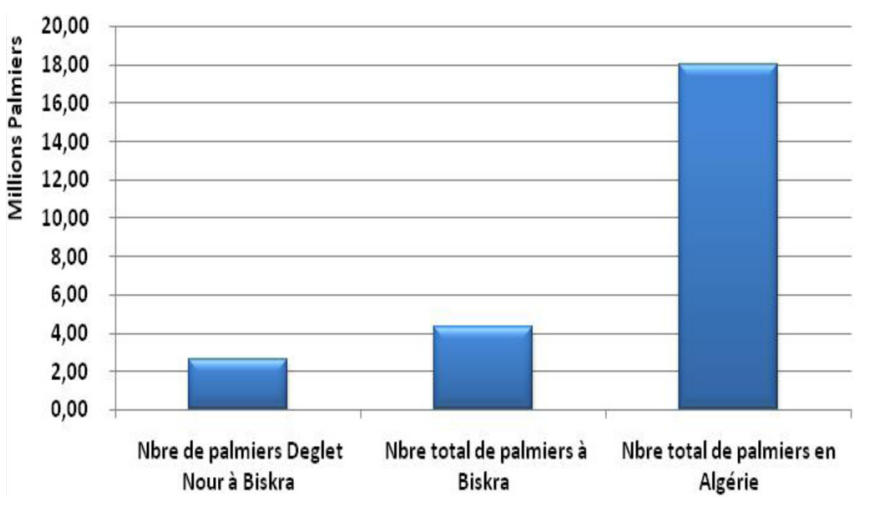

Fig. 2. Position du patrimoine phoenicicole de Biskra en 2015.

Fig. 2. Position of the trees date palm of Biskra in 2015.

opportunités qui existent sur le marché international des produits bio, qui est en expansion continue.

\section{Résultats et discussions}

\section{1 État des lieux et performances technico- économiques des dattes biologiques}

\subsection{1 État des lieux}

Bien que l'Algérie ait décidé en 2000 d'introduire et de promouvoir l'AB à travers les différents programmes de développement agricole, son application à la phoeniciculture

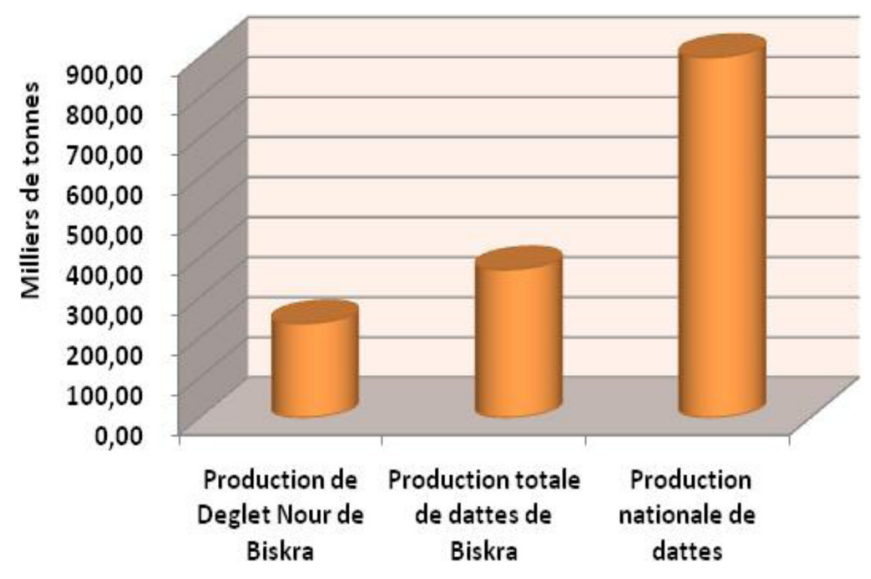

Fig. 3. Position de la production des dattes de Biskra en 2015.

Fig. 3. Position of date production of Biskra in 2015.

n'a commencé que depuis 2004. Les résultats obtenus restent en deçà des attentes (augmenter la superficie et la production des dattes bio, mais aussi le nombre des exploitations intégrées), eu égard aux moyens mis en place (diverses formes avantageuses de crédit agricole, subvention à l'exportation), et au regard des résultats très positifs enregistrés en Tunisie qui est plus avancée dans ce domaine (Rached et al., 2012).

Une analyse a été effectuée sur les principaux indicateurs techniques et économiques généralement utilisés pour évaluer les performances de l'AB (superficie, production, nombre de 
Tableau 1. Performance technique des dattes bio à Biskra en 2015.

Table 1. Technical performance of organic dates in Biskra in 2015.

\begin{tabular}{|c|c|c|c|c|c|}
\hline & & $\begin{array}{l}\text { Total } \\
\text { Palmier }\end{array}$ & $\begin{array}{l}\text { Palmier en } \\
\text { production }\end{array}$ & $\begin{array}{l}\text { Palmier } \\
\text { Deglet Nour }\end{array}$ & $\begin{array}{l}\text { Palmier Deglet Nour } \\
\text { en production }\end{array}$ \\
\hline Total palmier Biskra & (1) & 4286354 & 3894898 & 2638253 & 2334566 \\
\hline Superficie phoencicole de Biskra en ha & (3) & 28576 & 25966 & 17588 & 15564 \\
\hline Superficie phoencicole des 6 communes en ha & (4) & 6571 & 6395 & 5266 & 5107 \\
\hline Palmier biologique des 6 communes (12 800 pieds) & $(5)$ & & & & \\
\hline$\%$ superficie biologique & $(6) /(4)$ & 3,04 & 3,13 & 3,80 & 3,92 \\
\hline$\%$ superficie biologique & $(6) /(3)$ & 0,70 & 0,77 & 1,14 & 1,29 \\
\hline$\%$ palmier biologique & $(5) /(1)$ & 0,30 & 0,33 & 0,49 & 0,55 \\
\hline$\%$ palmier biologique & $(5) /(2)$ & 1,30 & 1,33 & 1,62 & 1,67 \\
\hline
\end{tabular}

${ }^{a}$ Tolga, Bouchagroune, Bordj ben azouz, Luchana, Foghala et Elghrous.

Source : calculé à partir des statistiques de la DSA de Biskra 2015.

producteurs bio, niveau de maîtrise des pratiques culturales et normes préconisées par la certification). Les résultats montrent que la région de Biskra est la plus importante au niveau national en matière de production et de commercialisation des dattes biologiques. Une progression, bien que variable d'un paramètre à un autre, a été enregistrée pour tous les indicateurs analysés.

Actuellement, dans la région, la superficie consacrée aux dattes certifiées bio ne dépasse pas 200 ha (Associations des producteurs des dattes de Biskra, 2015), mais elle s'étend d'année en année. Elle n'était que de 50 ha en 2004, avec un patrimoine de 12800 palmiers. Le nombre de producteurs reste faible (une vingtaine) mais ils n'étaient que sept à tenter l'aventure en 2004. Quant à la production, elle a augmenté de $200 \%$ en l'espace de 12 ans, pour atteindre $900 \mathrm{t}$, avec une prévision de 1500 t pour 2018, vu le nombre d'exploitations (près de 80 ) qui sont en conversion vers le bio et qui seront certifiées dans une ou deux années. Ce volume ne représente que $3 \%$ du tonnage des dattes exportées en 2015 et seulement $0,1 \%$ des $900000 \mathrm{t}$ de production nationale de la même année. Même si la datte bio est le produit phare de l'exportation de produits bio en Algérie, la phoeniciculture bio cible uniquement le cultivar Deglet Nour, à haute valeur marchande, et marginalise d'autres cultivars moins connus mais qui mériteraient cette forme de valorisation.

La pratique de la culture bio est présente dans plusieurs communes de l'ouest de la wilaya, notamment les communes de la daïra de Tolga, meilleur terroir pour cette variété de dattes, en raison des caractères pédoclimatiques de cette zone.

La superficie destinée à la production bio reste dérisoire (3\%) dans la superficie phoenicicole totale de ces communes. Elle l'est aussi par rapport à la superficie occupée par les palmiers en production $(3 \%)$, comme par rapport à la superficie productive consacrée à la seule Deglet Nour $(4 \%)$. Du point de vue patrimoine phoenicicole, la pratique bio ne touche que $1,3 \%$ des palmiers des communes concernées et $1,3 \%$ des palmiers en production, mais $1,7 \%$ du cultivar Deglet Nour (Tab. 1). Cette pratique culturale reste aussi embryonnaire par rapport au nombre de phoeniciculteurs de la région. Cependant, avec près de 640 palmiers, la propriété moyenne par producteur est importante, alors que la taille moyenne des exploitations de la région ne dépasse pas 350 palmiers et que les palmeraies connaissent un morcèlement continu lié aux successions (Benziouche et Cheriet, 2012).

\subsubsection{Performances technico-économiques de la culture bio des dattes}

L'étude de certains indicateurs techniques et économiques (production, rendement, superficie, chiffre d'affaires) pour 10 exploitations produisant des dattes certifiées bio dans la région, montre que la quasi-totalité $(90 \%)$ des producteurs certifiés interrogés ont enregistré une régression du rendement et de la qualité, ainsi qu'un manque à gagner estimé entre 25 et $32 \%$ en moyenne par rapport aux performances obtenues en phoeniciculture conventionnelle. Les causes sont multiples. La plus importante est l'application de l'itinéraire technique spécifique à l'AB, qui supprime ou modifie certaines pratiques culturales, notamment la fertilisation minérale et l'utilisation des pesticides. Seuls les produits naturels et les techniques non polluantes sont autorisés. Cependant, tous les producteurs déclarent qu'ils sont conscients de l'importance de l'AB et que cette diminution du rendement est largement compensée par le prix des dattes bio $(700 \mathrm{DA} / \mathrm{kg}$, soit $5,95 € / \mathrm{kg})$ correspondant à plus du double du prix payé pour les dattes ordinaires (250 à $300 \mathrm{DA} / \mathrm{kg}$, soit 2,13 à $2,55 € / \mathrm{kg}$ ). D'après Kenny et Hanafi (2001), d'une façon générale les rendements sont faibles dans la phase de reconversion, mais peu après et au fur et à mesure que l'environnement agrobiologique de la parcelle se stabilise, et que le producteur affine ses interventions agronomiques, les rendements doivent théoriquement s'améliorer, même s'ils n'atteignent jamais les rendements obtenus en culture conventionnelle. En outre, sur le plan agronomique, il existe actuellement plusieurs techniques bien adaptées au bio, et qui permettent d'améliorer les rendements. C'est le cas de la fertilisation, par l'apport de matière organique sous forme de fumier, d'engrais vert, de compost à base de palmes sèches et d'autres engrais naturels. Pour les traitements phytosanitaires, il s'agit de la combinaison entre des mesures préventives physiques non polluantes (désherbage manuel et mécanique) et des moyens de lutte biologique comme les bio-pesticides (Kenny et Hanafi, 2001). 
Seuls $20 \%$ des phoeniciculteurs certifiés $\mathrm{AB}$ affirment s'être réorientés vers l'AB pour des motifs économiques, notamment la garantie du débouché et l'amélioration du revenu. Ils sont un peu plus nombreux ( $30 \%$ des certifiés) à justifier le changement par la réduction attendue des charges productives grâce à la suppression de certaines pratiques culturales (apports de fertilisants chimiques, traitements phytosanitaires), pratiques exigées par la phoeniciculture conventionnelle mais bannies en AB.

Par ailleurs, la moitié des producteurs certifiés de dattes bio expliquent leurs choix par l'importance de l'AB dans la protection de l'environnement et pour le développement durable. En effet, beaucoup d'entre eux $(70 \%)$ ont déjà converti une grande partie de leurs propriétés en culture bio et le reste est en voie de conversion. Par contre, les autres (30\%) sont dans une phase d'essai et n'ont converti au bio qu'une faible part de leur patrimoine. Ils attendent de mesurer les résultats obtenus (amélioration du revenu), soit à partir de leurs propres essais, soit à partir de ceux de leurs voisins pratiquant la méthode bio.

La maitrise et le respect de l'itinéraire technique spécifique au bio sont variables d'un producteur à l'autre, d'une pratique à une autre, mais aussi d'une commune à l'autre, selon l'importance technico-économique de la pratique (comme la pollinisation et l'irrigation), son coût et l'état financier de l'exploitation (Benziouche et Chehat, 2010). Globalement, la maitrise est acceptable et tous les producteurs essayent d'appliquer convenablement les pratiques et les conseils reçus des institutions techniques (Institut technique de développement de l'agriculture saharienne et Institut national de protection des végétaux) ou de la part des vulgarisateurs. Toutefois, la plupart des conseils viennent des exportateurs (BIONOOR, BIODATTES et autres), ou des inspecteurs des instituts de certification bio (souvent ECOCERT) lors des inspections. Selon Hadjou et al. (2013), les premières exploitations qui ont réussi leur conversion au bio ont eu recours à des organismes de certification étrangers.

\subsection{Les contraintes de la production des dattes bio à Biskra}

Les obstacles qui empêchent un développement rapide de la production des dattes bio dans les Ziban, et de façon plus générale au niveau national, sont nombreux et de plusieurs ordres. En aval, c'est la déficience des exportations de ces produits bio vers les marchés solvables et porteurs, notamment européens. À l'instar de ce qui est vécu pour l'exportation des dattes conventionnelles, plusieurs causes expliquent cette déficience (Fig. 4) : la faiblesse des volumes proposés et la qualité des dattes, la faible compétitivité du produit, le fardage (fraude consistant à dissimuler dans les couches inférieures du colis des dattes de qualité moindre et de calibre différent de celles qui sont visibles et spécifiés par le marquage) pratiqué par certains exportateurs, l'insuffisance des moyens de transport maritime et aérien et les lenteurs des procédures administratives (Bouguedoura et al., 2015). À cela, s'ajoute la difficulté de pénétrer les marchés du bio, généralement contrôlés par des sociétés spécialisées, selon les exportateurs interrogés. Par ailleurs, $80 \%$ de nos producteurs certifiés bio déclarent comme contrainte,

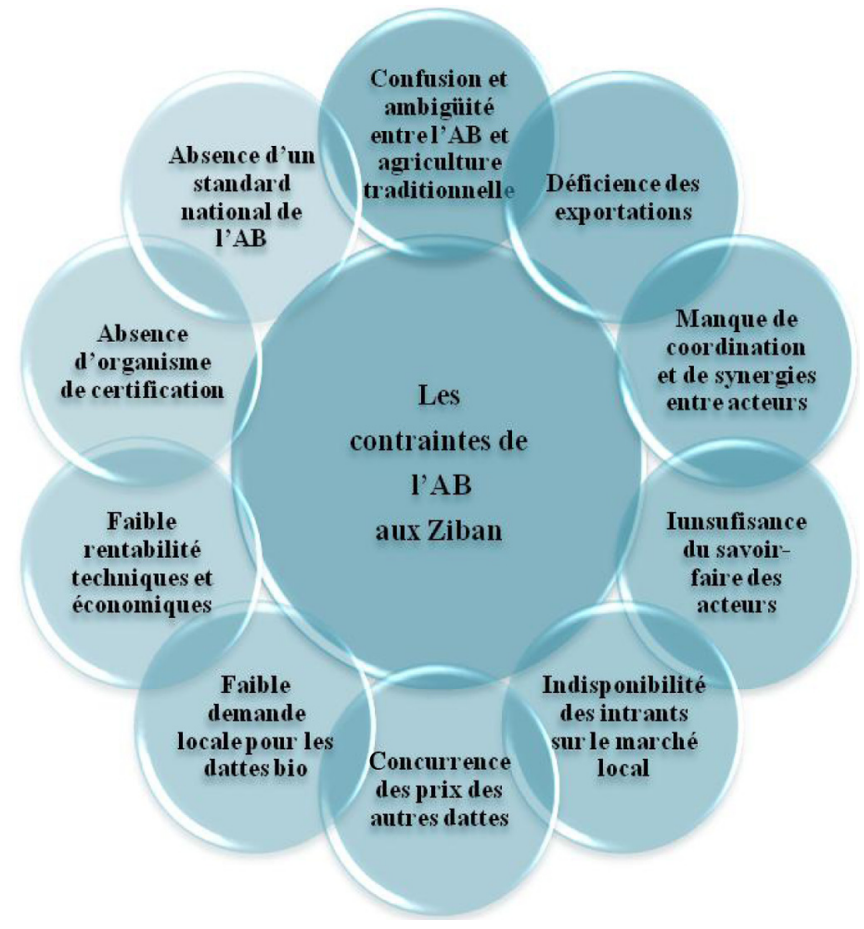

Fig. 4. Principales contraintes de la production des dattes bio au Ziban.

Fig. 4. Major constraints in the production of organic dates at Ziban.

l'absence quasi totale d'une demande locale ou nationale de dattes biologiques; certains rencontrent des difficultés pour commercialiser leurs productions dans de bonnes conditions, ce qui ne leur permet pas toujours de compenser leurs coûts. En effet, seuls $20 \%$ des producteurs ont été parfois sollicités par une demande locale de dattes bio. Cela prouve l'absence de toute démarche marketing et d'effort de sensibilisation des consommateurs algériens pour les intéresser aux produits bio, démarche nécessaire en l'absence d'une culture de consommation bio chez les Algériens (Benziouche et Abdelaoui, 2016).

De plus, il y a aussi la concurrence des autres dattes en termes de prix. Le prix relativement élevé des dattes bio $(10 € / \mathrm{kg}$ sur le marché européen et $700 \mathrm{DA} / \mathrm{kg}$, soit près de $6 € / \mathrm{kg}$ au niveau de l'exploitation) freine leur diffusion auprès des consommateurs. En outre, ces consommateurs préfèrent toujours la qualité morphologique (taille de la datte, longueur, diamètre, poids, couleur), plus présente dans les dattes conventionnelles que dans les dattes bio. Ce déficit de demande bio spécifique oblige parfois certains producteurs bio ( $10 \%$ de l'échantillon) à vendre leurs dattes au même prix que celles provenant de la culture conventionnelle.

En amont, la nécessité d'un soutien technique et d'une formation a été constatée dans les exploitations de l'enquête. En effet, la totalité des producteurs n'ont jamais bénéficié d'une formation à l' $\mathrm{AB}$. Les producteurs bio réclament ainsi un service de l'AB au niveau local et un réseau qui s'occuperait de la coordination des principaux acteurs de la filière pour sensibiliser les producteurs et diffuser le savoirfaire relatif aux pratiques de l'AB. Caplat (2012) souligne que la réussite de la conversion vers l'AB exige de bénéficier d'un 
soutien et d'un accompagnement technique qui commence par la formation.

Une autre contrainte est mise en relief par $30 \%$ des producteurs : le coût élevé et parfois même la non-disponibilité, en quantité et en qualité, de certains fertilisants organiques et bio-pesticides (Biomite) sur le marché local (Fig. 4).

Nos investigations ont permis de cerner d'autres contraintes de l'AB en Algérie, valables pour la production des dattes bio:

- le manque de savoir-faire des agriculteurs et des institutions ;

- l'absence de sensibilisation et le manque de coordination et de synergie entre les différents intervenants (Abdellaoui, 2012);

- parfois, la confusion et l'ambiguïté entre l'agriculture traditionnelle et biologique;

- l'absence d'un standard national de l'AB;

- l'absence d'organismes internationaux de certification à l'échelle nationale (Abdellaoui, 2013);

- la difficulté d'application des textes réglementaires relatifs aux produits agricoles de qualité.

Cependant, la contrainte majeure reste la faible rentabilité économique de la production de dattes bio. En effet, plusieurs producteurs de dattes bio ont enregistré des performances économiques décevantes après l'application des techniques bio (Fig. 4). Si $60 \%$ des producteurs ont amélioré leur chiffre d'affaires après l'introduction du bio, $30 \%$ n'ont enregistré aucune amélioration, alors que $10 \%$ ont constaté une diminution de ce chiffre d'affaires, de 10 à $15 \%$ en moyenne. Cette réduction du chiffre d'affaires ne s'explique pas uniquement par la pratique de l'AB; dans certains cas, les résultats tardent à voir le jour, ce qui décourage les producteurs et les conduit parfois à l'abandon de ce mode de production, car ils le considèrent peu performant, même si les résultats enregistrés dans d'autres palmeraies et pour d'autres cultures, au niveau national et dans les pays européens, indiquent l'inverse. Les faibles rendements enregistrés par cette minorité de producteurs de dattes bio peuvent s'expliquer soit par les contraintes rencontrées (notamment naturelles), soit par la non-maîtrise de la conduite propre à la culture bio. Par exemple, l'agriculteur se base sur le fumier et néglige la pratique des engrais verts et le recours aux engrais naturels autorisés par la réglementation (Kenny et Hanafi, 2001).

\subsection{Les obstacles à la conversion en bio et à l'adhésion à la pratique du bio}

Pour certains producteurs, le passage à l'AB implique beaucoup de contraintes (Fig. 5). Ils ont donc des difficultés à respecter les techniques spécifiques à l'AB (confusion au début de la conversion) et considèrent, pour $60 \%$ des producteurs non certifiés (en cours de conversion), qu'il y une insuffisance de vulgarisation. D'autres producteurs, $20 \%$ du même échantillon, sont rebutés par le coût de production élevé, car ils considèrent qu'ils déploient énormément d'efforts et de moyens durant cette phase de conversion. Pour eux, tous les travaux se font manuellement, en plus des charges supplémentaires dues à l'utilisation de quelques outils et produits

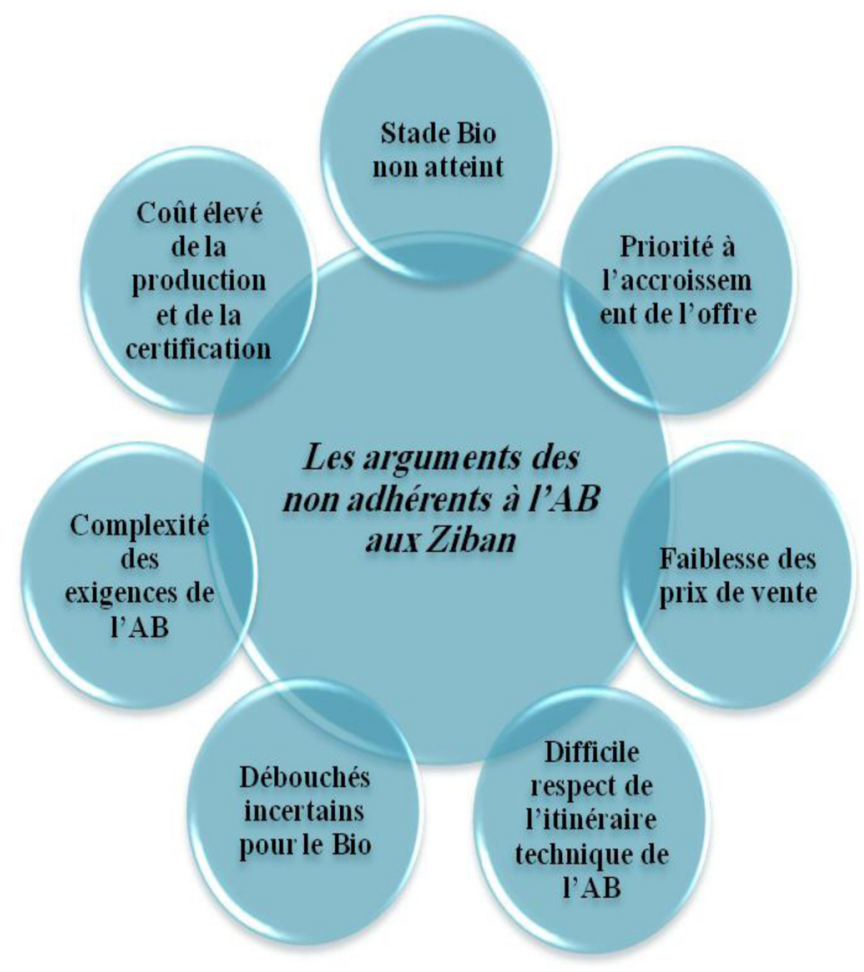

Fig. 5. Les arguments des non-adhérents à l'AB au Ziban.

Fig. 5. The arguments of non-practitioners in organic dates at Ziban.

spécifiques à la production de dattes bio et qui ne bénéficient d'aucune subvention. Cela diminue la compétitivité des exploitations et influe parfois négativement sur leurs performances économiques. Selon $10 \%$ des producteurs en cours de conversion au bio, ces contraintes se conjuguent au risque toujours présent de contamination, en raison de l'utilisation des pesticides dans les palmeraies limitrophes. Effectivement, l'application de l'AB dans les palmeraies paraît plus difficile que son application pour d'autres cultures, dans la mesure où les palmeraies bio, intégrées dans une oasis, sont forcément limitrophes de palmeraies non bio ; les exploitations phoenicicoles isolées sont très rares.

De ce fait, la contamination est un risque important. Il est impossible d'empêcher le chevauchement du système racinaire des palmiers entre les deux types d'exploitations (Fig. 5) ni d'éviter la contamination en cas de traitement phytosanitaire par pulvérisation dans les palmeraies non bio (diffusion éolienne). Ce risque de contamination s'élargit avec l'extension du maraîchage dans la région (plus de 4000 ha), pour lequel l'utilisation non raisonnée des pesticides et des insecticides est flagrante. D'ailleurs, c'est aussi l'un des arguments avancés par plusieurs des phoeniciculteurs conventionnels interrogés $(20 \%)$ pour expliquer la non-introduction de l'AB dans leurs palmeraies.

D'autres parmi eux (10\%) avancent l'argument de la difficulté, de la complexité et de l'exigence de cette agriculture. Le troisième argument avancé est l'absence de débouchés pour les dattes bio. Certains $(10 \%)$ considèrent que l'AB ne peut concerner que les pays où il $\mathrm{y}$ a une forte utilisation de produits chimiques. Pour eux, les dattes de la région sont produites généralement dans des conditions 


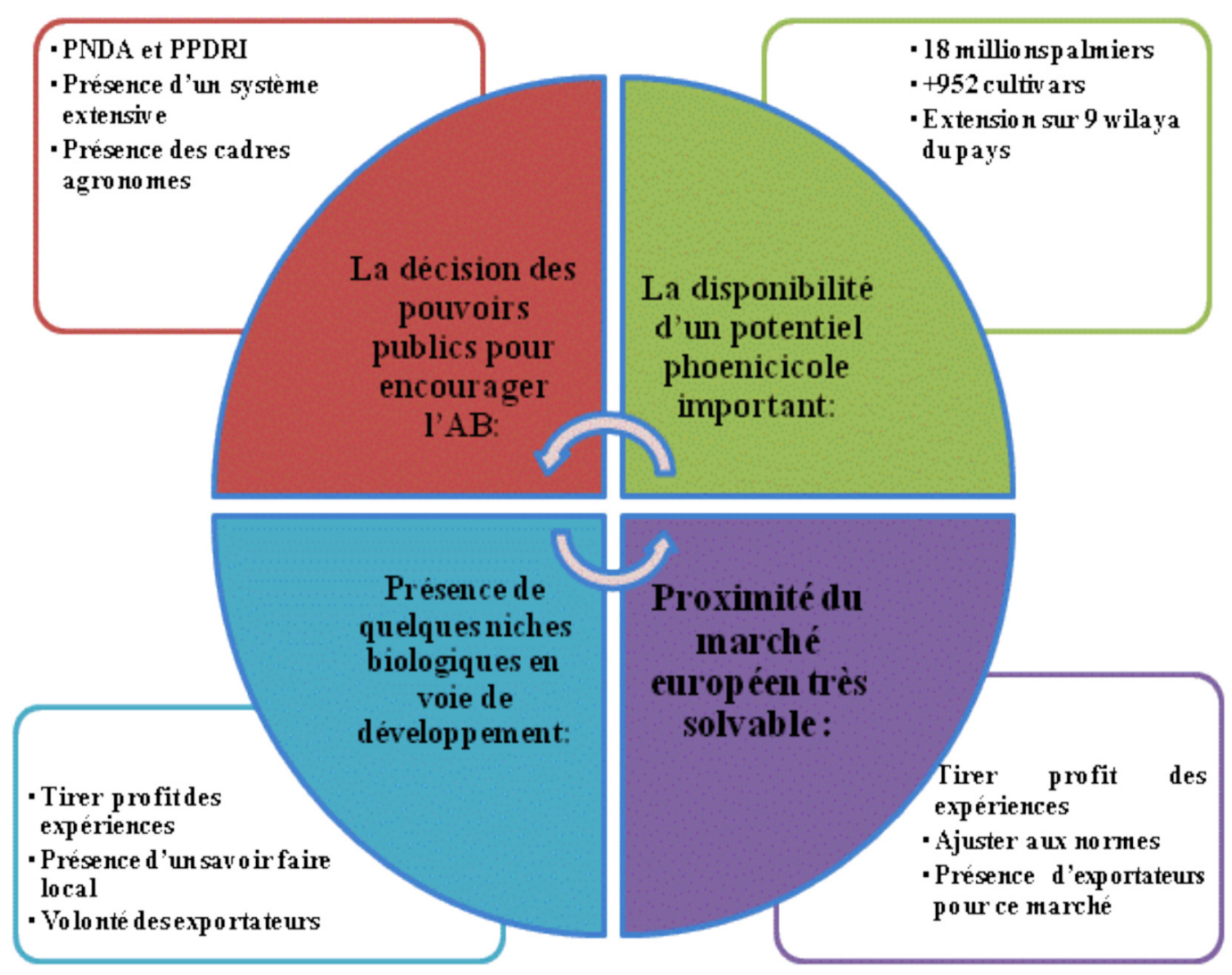

Fig. 6. Opportunités et facteurs dirigeants pour le développement de l'AB des dattes de Ziban.

Fig. 6. Opportunities and factors for the development of organic agriculture on Ziban dates.

extensives et peuvent être assimilées à des produits bio (Abdellaoui, 2013). Il n'en reste pas moins que pour la majorité des producteurs $(60 \%)$, il faut donner la priorité à l'accroissement des volumes de production, car selon eux, le stade de la production bio, production «de luxe», n'est pas encore atteint (Fig. 5). De plus, disent-ils, la demande pour les dattes conventionnelles est en croissance continue et l'offre ne suit pas suffisamment, ce qui limite l'intérêt de la conversion au bio.

Il faut par ailleurs souligner que, durant l'enquête, nous avons constaté fréquemment une confusion et une ambiguité entre agriculture bio et conventionnelle; il est fréquent, en effet, d'entendre dire que l'AB est conventionnelle. Alors que selon Caplat (2012), l'AB ne peut pas se résumer en la suppression des produits chimiques de synthèse; les règles de production vont bien au-delà et imposent une réorganisation profonde des systèmes organiques.

\subsection{Opportunités et atouts pour le développement des dattes bio}

Pour la filière dattes dans la région des Ziban et en Algérie, les éléments de succès existent et les opportunités sont présentes, mais faiblement exploitées. On note en effet la disponibilité d'un potentiel phoenicicole important et diversifié, avec une possibilité de production précoce et tardive de certains cultivars ainsi que des conditions pédoclimatiques très favorables à l'AB (Fig. 6). Le système de culture actuel reste extensif, la mécanisation est totalement absente et l'utilisation des intrants chimiques est faible dans les exploitations, ce qui favorise une conversion vers le bio. De plus, dans cette région, on trouve un savoir-faire local et traditionnel qui mérite d'être capitalisé et exploité. La troisième opportunité est la présence, dans certains systèmes de production, de quelques niches biologiques en voie de développement (Abdellaoui, 2013) dont l'expérience pourrait être diffusée.

La décision des pouvoirs publics, à travers plusieurs décrets, d'encourager l'AB par différents programmes (Programme national de développement agricole « $2000 »$, Programme national de développement agricole et rural «2003») et Politique du renouveau agricole et rural (2013), constitue une opportunité. Par ailleurs, la proximité du marché européen très solvable, pourrait être mieux exploitée par les producteurs bio, en tirant profit des expériences européennes dans le domaine pour mieux s'ajuster aux normes internationales (Fig. 6). Elle permettrait également aux exportateurs de pénétrer ces marchés et/ou de renforcer leurs parts de marché en profitant de l'Accord d'association avec l'UE, dans le cadre d'échanges multilatéraux (Abdellaoui, 2013).

Une autre opportunité, non moins importante que les précédentes, s'offre aux producteurs, c'est l'existence d'un avantage compétitif sur le marché de l'export et la volonté de certains exportateurs de promouvoir l'exportation des produits 
bio. L'expérience des deux entreprises d'exportation (BIODATTE et BIONOOUR) est un bon exemple. C'est pour cela que plusieurs exportateurs se sont convertis au bio. Enfin, on notera l'existence de plusieurs agronomes formés dans ce sens qui pourraient sans doute renforcer le savoir local (Fig. 6).

Enfin, il s'agit d'exploiter l'opportunité offerte par la forte croissance de la demande sur le marché bio à l'échelle mondiale (15 milliards \$US en 1999 et 80 milliards \$US en 2013) (Mercier, 2015) et l'importance des prix offerts par rapport au conventionnel, en tirant le meilleur parti possible de la mise en application du label «Deglet Nour de Tolga» à partir de 2017.

\section{Conclusion}

L'AB joue un rôle important dans le développement local de nombreux pays et particulièrement pour la phoeniciculture dans certains pays voisins qui n'ont pas autant de potentialités agronomiques que l'Algérie. La Tunisie, à titre d'exemple, a fait de grands progrès en $\mathrm{AB}$. Elle est actuellement un pays leader dans la production et l'exportation des dattes bio. Elle a produit et exporté $5780 \mathrm{t}$ de dattes biologique en 2015 et près de $6000 \mathrm{t}$ en 2016 , ce qui représentait $7,8 \%$ du total de ses exportations de dattes durant cette année. Ce mode de production y a connu une évolution très importante depuis 2000 , date à laquelle la production ne dépassait pas $500 \mathrm{t}$ (GFRuit, 2016).

Notre étude a cependant montré que l'application des techniques de l'AB à la phoeniciculture dans la région des Ziban, tout comme pour d'autres cultures en Algérie, reste à l'heure actuelle sous-exploitée, peu organisée, peu encadrée et pour laquelle l'information reste très fragmentée. Ceci s'explique par la conjugaison de plusieurs contraintes, d'ordre technique, socioéconomique et institutionnel, qui entravent le développement de la pratique de l'AB. En effet, la plupart des indicateurs demeurent peu performants et ce, en dépit des opportunités nombreuses et encourageantes qui se trouvent dans l'environnement politique, économique, technique et social.

Ces opportunités devraient être exploitées convenablement à tous les niveaux afin de développer et promouvoir cette filière bio dans le pays. Ceci ne réussira pas sans donner à ce mode de production la place qu'il mérite par la mise en application rigoureuse des textes réglementaires, en tirant profit des expériences des pays plus avancés dans ce domaine. La mise en place d'un réseau national reliant les principaux acteurs de la filière $A B$ et l'installation d'un service technique au niveau local constituent aujourd'hui deux nécessités. Cela permettrait d'assurer un accompagnement de proximité des producteurs pour la maîtrise des itinéraires techniques et donc l'amélioration des rendements. Cela permettrait aussi de renforcer la coordination entre les acteurs et de promouvoir une stratégie claire pour l'extension de l'AB.

\section{Références}

Abdellaoui H. 2012. Développement récent et perspectives de l'agriculture biologique en Algérie. In : Colloque international sur les produits de terroir, université de Blida.

Abdellaoui H. 2013. Présentation de l'approche algérienne en matière d'AB. In : Colloque international sur la valorisation des produits agricoles. Mostaganem : ministère de l'Agriculture.

Belguedj A. 2004. Analyse diagnostique du secteur du palmier dattier en Algérie : étude des marchés des produits du palmier dattier au Maghreb. Étude PNUD.

Benziouche SE. 2012. Analyse de la filière dattes en Algérie; constats et perspectives de développement. Cas de la daïra de Tolga. Thèse de doctorat ès agronomie. Alger: ENSA, $470 \mathrm{p}$.

Benziouche SE, Chehat F. 2010. La conduite du palmier dattier dans les palmeraies des Ziban (Algérie). Quelques éléments d'analyse. Rev EJSR 42: 644-660.

Benziouche SE, Cheriet F. 2012. Structures et contraintes de la filière dattes en Algérie. Rev New Medit 4: 49-57.

Benziouche SE, Abdelaoui I. 2016. Rôle de la consommation dans la valorisation des produits de terroirs, cas de la Deglet Nour Tolga. In : Workshop international sur la durabilité des systèmes de production phonicicoles en Algérie, université de Biskra.

Bouguedoura N, Bennaceur M, Babahani S, Benziouche SE. 2015. Date palm status and perspective in Algeria. In: Al-Khayri JM, Jain SM, Jhnson DV, eds. Date Palm Genetic Resources and Utilization. Volume 1: Africa and the Americas. Germany: Springer, pp. 125-168.

Caplat J. 2012. L'agriculture biologique pour nourrir l'humanité. France: Actes sud, 479 p.

Cardona A. 2014. Le développement de l'agriculture biologique : effets directs et indirects dans le monde agricole et non agricole. Une enquête en Île-de-France. Eco Rur 339: 183-194.

Direction des services agricoles (DSA) Biskra. 2016. Rapport : situation de l'agriculture de la wilaya de Biskra.

Georges I, Blanc J. 2012. L'émergence de l'agriculture biologique au Brésil : une aubaine pour l'agriculture familiale ? Le cas de producteurs de la ceinture verte de la ville de São Paulo. Autrepart 64: 121-138. DOI: 10.3917/autr.064.0121.

GFRuit. 2016. Statistiques du groupement interprofessionnel des fruits tunisien. www.GFRuit.com. Consulté le 25/12/2016.

Hadjou L, Cheriet F, Djenane A. 2013. Agriculture biologique en Algérie : potentiel et perspectives de développement. Cah CREAD 105: 113-132.

Kenny L, Hanafi A. 2001. L'agriculture biologique au Maroc. Bulletin d'information du PNTTA (IAV), Transfert de technologie en agriculture 82: 1-4.

Mercier E. 2015. Organic Farming's Panorama Worldwide, in the European Union and France: What movements in depth? $10^{\text {ème }}$ Séminaire international sur l'agriculture biologique. Paris, 26 février 2015.

Peigné J. 2015. Évaluation participative de prototypes de systèmes de culture combinant agriculture de conservation et agriculture biologique. Cah Agric 24: 134-141.

Rached Z, Salmi A, Khaldi R. 2012. Les performances techniques des dattes biologiques et conventionnelles en Tunisie : cas de la région de Hezoua. Rev New Medit 3: 50-58.

Citation de l'article : Benziouche SE. 2017. L'agriculture biologique, un outil de développement de la filière dattes dans la région des Ziban en Algérie. Cah. Agric. 26: 35008. 